\section{JTI}

JOURNAL OF

TRAUMA AND INJURY

Received: May 3, 2021

Revised: June 22, 2021

Accepted: June 28, 2021

\section{Correspondence to}

Chan Yong Park, M.D., Ph.D.

Division of Trauma Surgery, Department of Surgery, Seoul National University Hospital, 101 Daehak-ro, Jongno-gu, Seoul 03080, Korea

Tel: $+82-2-2072-2817$

Fax: +82-2-766-3975

E-mail: trauma-park@naver.com

ORCID: https://orcid.org/0000-00025111-3270

\title{
Non-Operative Management with Angioembolization of Grade IV and V Renal Injuries in a Hybrid Emergency Room System
}

\author{
So Ra Ahn, M.D. ${ }^{1}$, Sang Hyun Seo, M.D. ${ }^{2}$, Joo Hyun Lee, M.D. ${ }^{1}$, \\ Chan Yong Park, M.D., Ph.D. ${ }^{3}$ \\ ${ }^{1}$ Department of Trauma Surgery, Wonkwang University Hospital Regional Trauma Center, \\ Iksan, Korea \\ ${ }^{2}$ Department of Radiology, Wonkwang University Hospital Regional Trauma Center, \\ Iksan, Korea \\ ${ }^{3}$ Division of Trauma Surgery, Department of Surgery, Seoul National University Hospital, \\ Seoul, Korea
}

Renal injuries occur in more than $10 \%$ of patients who sustain blunt abdominal injuries. Non-operative management $(\mathrm{NOM})$ is the established treatment strategy for lowgrade (I-III) renal injuries. However, despite some evidence that NOM can be successfully applied to high-grade (IV, V) renal injuries, it remains unclear whether NOM is appropriate in such cases. The authors report two cases of high-grade renal injuries that underwent NOM after embolization in a hybrid emergency room (ER) system with a 24/7 in-house interventional radiology (IR) team. A 29-year-old male visited Wonkwang University Hospital Regional Trauma Center complaining of right abdominal pain after being hit by a rope. Computed tomography (CT) was performed 16 minutes after arrival, and the CT scan indicated a grade V right renal injury. Arterial embolization was initiated within 31 minutes of presentation. A 56-year-old male was transferred to Wonkwang University Hospital Regional Trauma Center with a complaint of right flank pain. He had initially presented to a nearby hospital after falling from a 3-m height. Thanks to the key CT images sent from the previous hospital prior to the patient's arrival, angiography was performed within 8 minutes of the patient's arrival and arterial embolization was completed within 25 minutes. Both patients were treated successfully through NOM with angioembolization and preserved kidneys. Hematoma in the first patient and urinoma in the second patient resolved with percutaneous catheter drainage. The authors believe that the hybrid ER system with an in-house IR team could contribute to NOM and kidney preservation even in high-grade renal injuries.

Keywords: Renal injury; Non-operative management; High-grade; Hybrid emergency room system; Embolization 


\section{JTI}

\section{INTRODUCTION}

Renal injuries occur in more than $10 \%$ of patients who sustain blunt abdominal injuries. For decades, non-operative management (NOM) has been the established treatment strategy for low-grade (I-III) renal injuries; however, studies investigating the efficacy of NOM for high-grade (IV, V) renal injuries are limited. Despite some evidence that NOM can be successfully applied to high-grade renal injuries, it remains unclear whether this approach is appropriate [1] The decision to perform interventional or conservative treatment depends on each patient's overall treatment strategy and the circumstances of each hospital.

The regional trauma center of Wonkwang University Hospital operates a dual-room hybrid emergency room (ER) system (Fig. 1) with a continuously staffed, in-house interventional radiology (IR) team. The following cases

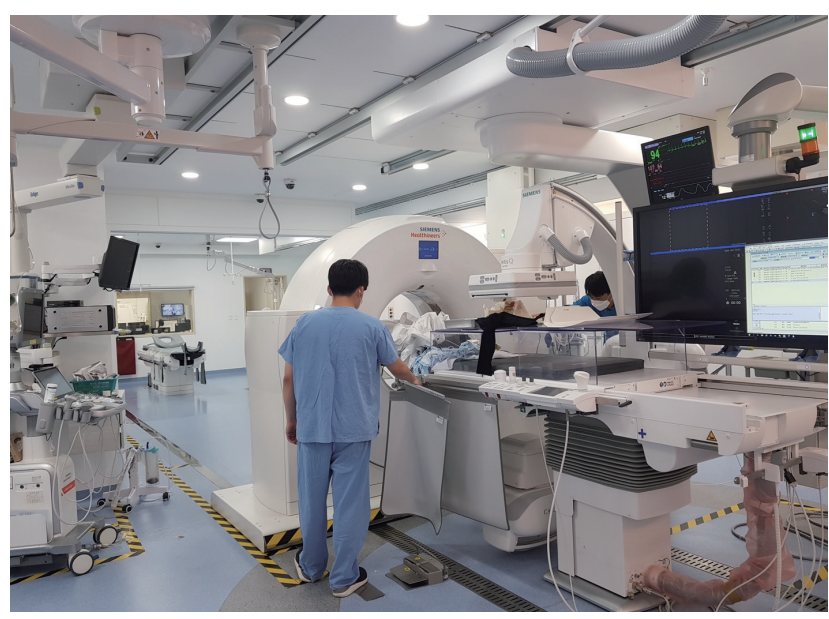

Fig. 1. Dual-room hybrid emergency room. describe the treatment of high-grade renal injuries within this system.

\section{CASE REPORT}

\section{Case 1. Grade $\mathbf{V}$ renal injury}

A 29-year-old male presented to Wonkwang University Hospital Regional Trauma Center with complaints of gross hematuria and right abdominal pain. The pain started after he sustained a blow to his right abdomen from a rope while working on a ship, approximately 90 minutes prior to being seen at the hospital. The patient was immediately transferred to the dual-room hybrid ER where a focused assessment with sonography in trauma was performed; the assessment conveyed positive findings. The patient's Glasgow Coma Scale (GCS) score was 15 and his initial vital signs were stable: blood pressure, 120/70 $\mathrm{mmHg}$; pulse, 61 beats/min. The patient demonstrated right lower abdominal tenderness, and his computed tomography (CT) results were available within 16 minutes of his arrival at the center. The chest CT results indicated fractures of ribs 10 and 11; however, the abdominopelvic CT scan indicated a grade $\mathrm{V}$ right renal injury and a massive retroperitoneal hematoma (Fig. 2). Contrast extravasation from the upper pole of the right kidney was observed, and the patient underwent emergency angiography. An ultrasound-guided, right common femoral artery puncture was performed 31 minutes after the patient's arrival. Extravasation from and multiple pseudoaneurysms in the superior segmental artery were identified using right renal arteriography. Following coil embolization, further bleeding was not observed on follow-up renal arteriography (Fig. 3). Since the patient's
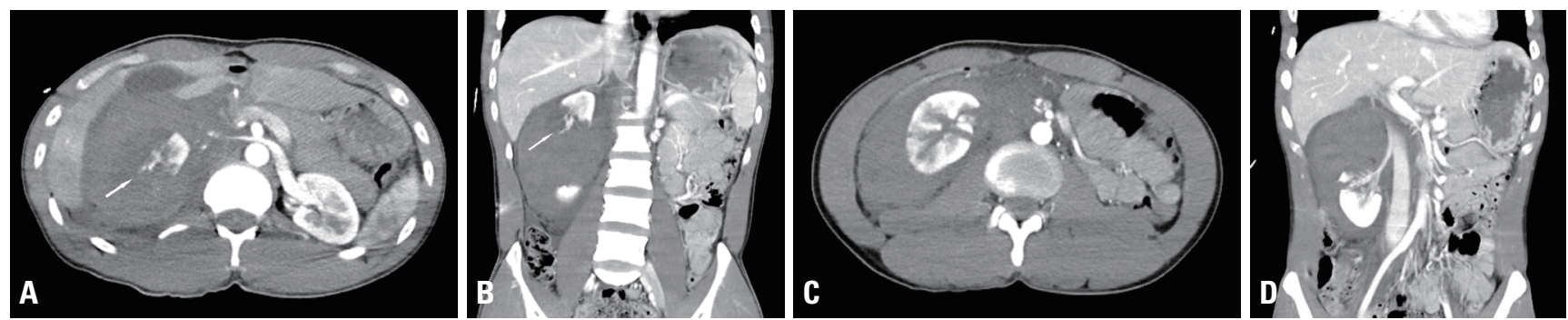

Fig. 2. (A-D) Abdominal computed tomography (axial and coronal images) of the patient with a grade $V$ injury, showing the separated vascularized kidneys and a hypodense hematoma. Contrast extravasation is seen in the upper pole of the right kidney (arrows of A, B). 
initial hemoglobin $(\mathrm{Hb})$ level was $12.0 \mathrm{~g} / \mathrm{dL}$ and his vital signs were stable, an emergency transfusion was not performed. His initial laboratory tests also showed blood concentrations of ALT $681 \mathrm{U} / \mathrm{L}$, AST $122 \mathrm{U} / \mathrm{L}$, creatine kinase $648 \mathrm{IU} / \mathrm{L}$, blood urea nitrogen (BUN) $25.0 \mathrm{mg} / \mathrm{dL}$, and creatinine $0.86 \mathrm{mg} / \mathrm{dL}$; urine microscopy revealed 1,000,000 red blood cells (RBCs)/high-power field (HPF). After embolization, the patient's follow-up $\mathrm{Hb}$ level was $11.9 \mathrm{~g} / \mathrm{dL}$, which was not significantly different from the initial level. Three days after embolization, active bleeding was not observed on the follow-up abdominopelvic CT scan. Although the right upper renal parenchyma was completely devascularized, the right inferior residual renal parenchyma showed contrast enhancement. Significant changes were not observed on a 1-week postadmission abdominopelvic CT scan. However, the patient complained of abdominal pain and exhibited abdominal distension due to the hematoma. Percutaneous catheter drainage (PCD) was performed to remove the perirenal fluid collection. After 3 weeks of hospitalization, the patient was discharged without any further complications. Six weeks after its insertion, the PCD catheter was removed in the outpatient
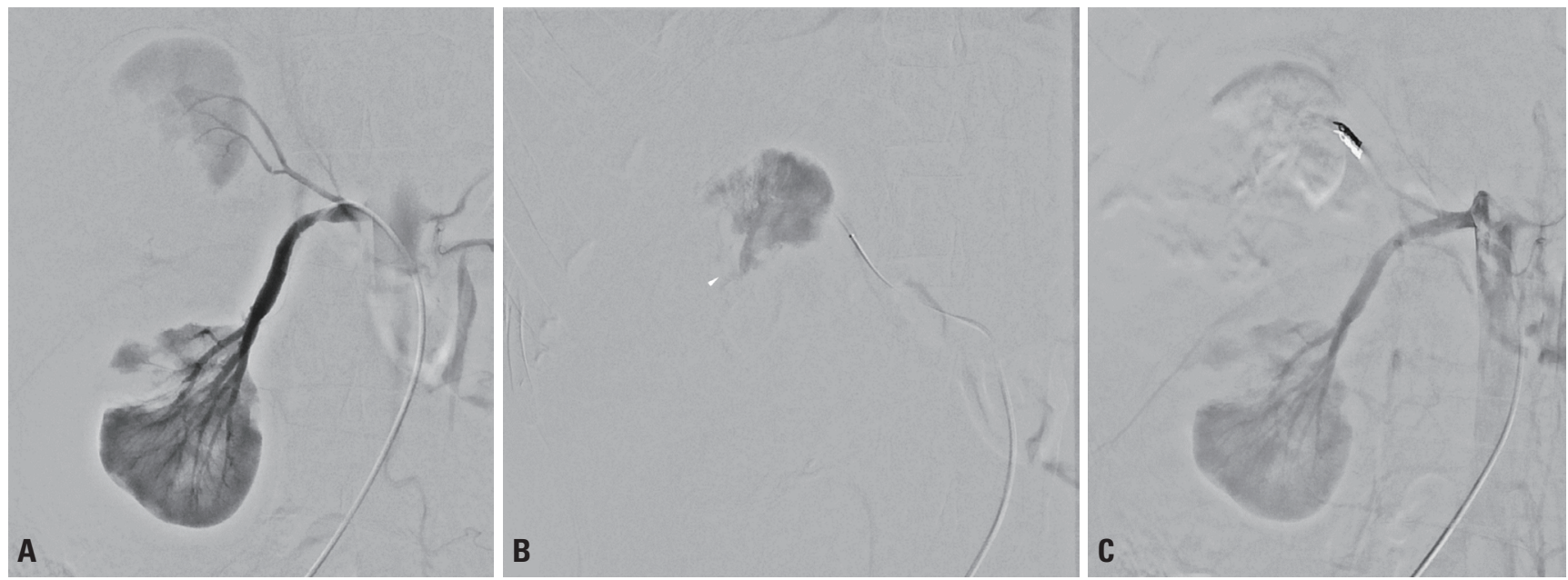

Fig. 3. (A) Right renal angiography of the patient with a grade $V$ injury shows separation of the upper and lower poles. (B) Contrast extravasation is seen during selective angiography (arrowhead). (C) The upper polar artery is shown after complete embolization using a coil.
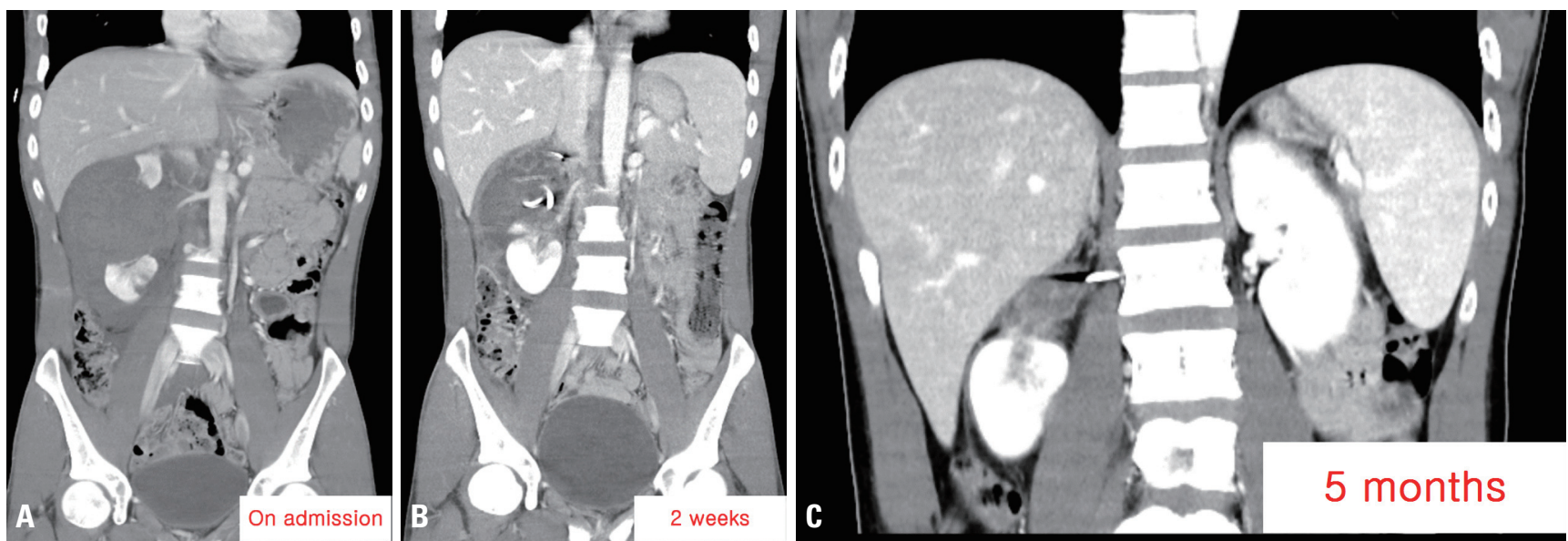

Fig. 4. (A-C) These computed tomography images show the postprocedural course of the patient with a grade $V$ injury. The large hematoma, shown in the upper pole of the right kidney, is observed to have gradually decreased in size. (C) Five months after the accident, the hematoma is no longer evident. 


\section{JTI}

clinic (Fig. 4). In this case, the patient required transfusion of only one unit of packed RBCs (pRBCs) and one unit of fresh frozen plasma (FFP) during hospitalization.

\section{Case 2. Grade IV renal injury}

A 56-year-old male was transferred to Wonkwang University Hospital Regional Trauma Center with complaints of right flank pain. He had initially present to a nearby hospital after falling from a 3-m height. There, he had undergone non-contrast-enhanced abdominopelvic CT that suggested severe right renal injury. During the patient's transfer to Wonkwang University Hospital Regional Trauma Center, the previous hospital was asked to provide a key CT image. This allowed the trauma team to review the image (Fig. 5) and prepare a radiologic intervention before the patient's arrival. As soon as the
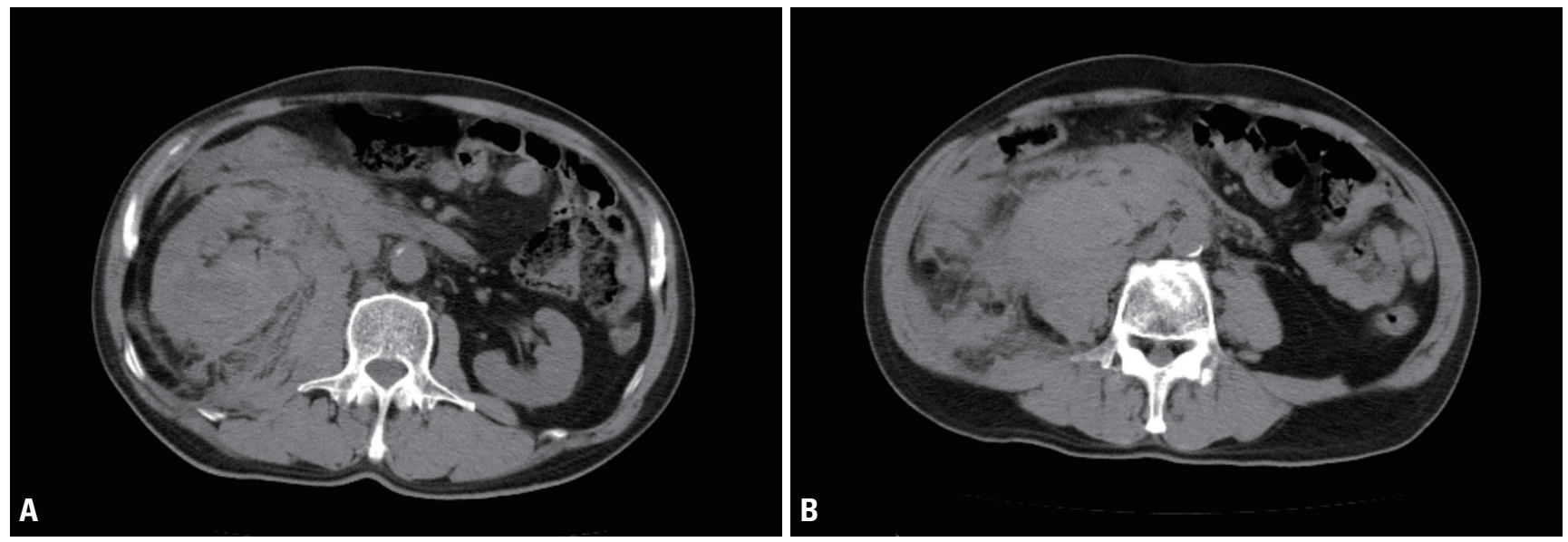

Fig. 5. (A) Abdominal non-contrast computed tomography (axial image) in the patient with a grade IV injury shows large right perirenal and pararenal hematomas. (B) A right psoas muscle hematoma and a retroperitoneal hematoma are also seen.
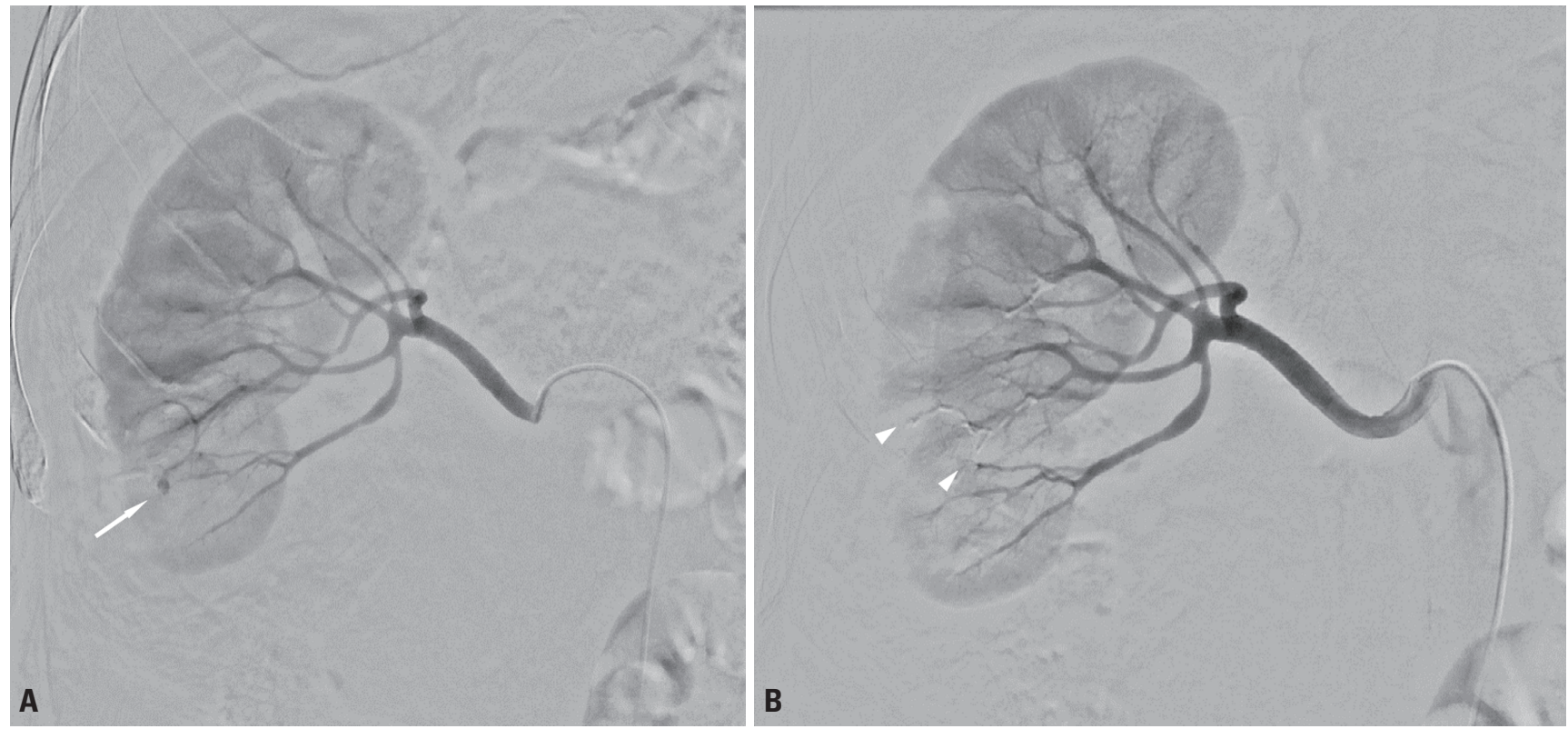

Fig. 6. (A) Right renal angiography of the patient with a grade IV injury shows a small pseudoaneurysm in an arcuate artery in the lower pole (arrow). (B) This image was obtained after injecting Histoacry ${ }^{\circledR}$ (B. Braun, Melsungen, Germany) and Lipiodol ${ }^{\circledR}$ (Jubilant HollisterStier, Spokane, WA, USA). Embolized blood vessels are seen in a lower polar artery (arrowheads). 
patient arrived, he was taken to the hybrid ER where his GCS score was 15 and his initial vital signs were unstable (blood pressure 76/48 mmHg; pulse 75 beats/min); his abdomen was soft and mildly distended but not tender. Ultrasound-guided right common femoral artery puncture was performed 8 minutes after the patient's arrival. Arteriography revealed extravasation from the lower polar artery of the right kidney and multiple extravasations from the right lumbar artery. Therefore, embolization was performed using Histoacryl ${ }^{\circledR}$ (B. Braun, Melsungen, Ger-

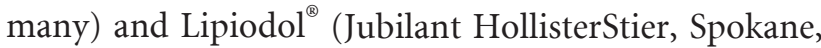
WA, USA) in the right lower polar artery and Gelfoam ${ }^{\circledR}$ (Pfizer, New York, NY, USA) particles in the right lumbar artery (Fig. 6). Postembolization, contrast-enhanced CT was performed, but focal bleeding was not observed. A grade IV right renal injury, a large hemoperitoneum, hemoretroperitoneum (Fig. 7A), and a left tibia fracture were identified. The patient's initial $\mathrm{Hb}$ level was $11.0 \mathrm{~g} / \mathrm{dL}$, but he was hemodynamically unstable; an emergency transfusion of two units of $\mathrm{O}^{+}$pRBCs was started within 2 minutes of his arrival. Ten minutes after presentation, his systolic blood pressure was $103 \mathrm{mmHg}$, and an additional two units of unmatched ABO type-specific pRBCs and four units of FFP were transfused; after the transfusion, his $\mathrm{Hb}$ level was $11.6 \mathrm{~g} / \mathrm{dL}$. The patient received a total of seven units of pRBCs and four units of FFP while he was hospitalized. Laboratory tests showed a mildly elevated BUN concentration $(21.0 \mathrm{mg} / \mathrm{dL})$ and a creatinine level
$(0.97 \mathrm{mg} / \mathrm{dL})$ that was within the normal range. Gross hematuria was observed during Foley catheterization, and urine microscopy showed 22,499 RBCs/HPF. Two days after embolization, the patient's hemoretroperitoneum had decreased according to the follow-up CT. However, the hematoma in the renal pelvis had increased in size, leading to suspicion of a collecting duct injury. However, the continued mild pain in the patient's right flank and the increasing size of the hematoma led to a suspicion of a collecting duct injury. Fortunately, the hemoretroperitoneum had sharply decreased in size on a follow-up CT scan performed 2 weeks after admission. Right periureteral fluid collection that was thought to be urinoma was observed around the psoas muscle, and a PCD was performed. After 1 week, the amount of fluid draining through the PCD had decreased, and the urine turbidity reduced and it became clear. Follow-up CT indicated that the fluid collection along the right psoas muscle had markedly decreased, and the PCD catheter was removed. The urinoma around the kidney was not present on follow-up CT performed 40 days after the accident (Fig. 7B-D), and the patient was discharged without further complications.

\section{DISCUSSION}

Renal injuries account for approximately $1-5 \%$ of all
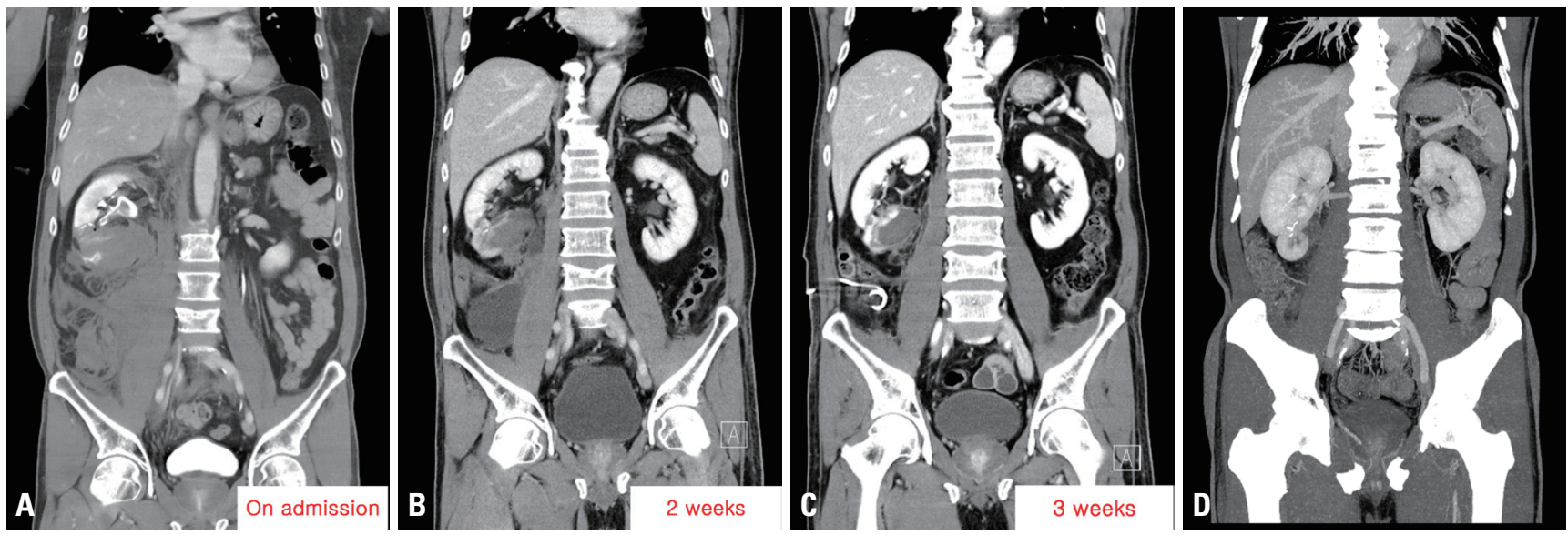

Fig. 7. (A) Immediately after the procedure in the patient with a grade IV injury, large hematomas are seen in the right perirenal space, pararenal space, and right psoas muscle. $(B, C)$ The hematomas are seen to gradually decrease in size, and percutaneous catheter drainage was performed during follow-up. (D) A maximum intensity projection image shows that the shape of the kidney is well maintained. 
trauma cases, and the kidney is the most commonly injured genitourinary organ [2]. Approximately 90-95\% of renal injuries are caused by blunt injuries [3], such as those caused by high-energy deceleration collisions (e.g., motor vehicle accidents, falls, and assaults) [2]. The most commonly used measure of renal injury is the American Association for the Surgery of Trauma (AAST) renal injury scale. The renal injury scale is divided into five grades, with grades IV and V usually being referred to as highgrade injuries [4]. According to the 2018 AAST revised grading, grade IV renal injuries include segmental vascular injuries, segmental or complete parenchymal infarctions, and active bleeding beyond the Gerota fascia; grade $\mathrm{V}$ injuries include main renal artery or vein lacerations, hilum avulsion, kidney devascularization with active bleeding, and shattered kidney with loss of identifiable parenchymal renal anatomy $[5,6]$.

Previously, intravenous urography and renal arteriography were used to evaluate hemodynamically stable patients [7]. Currently, however, abdominopelvic multi-detector CT (MDCT) with contrast enhancement is the imaging modality of choice for hemodynamically stable patients with genitourinary injuries. MDCT is fast, widely available, and provides anatomical and functional data [8].

During the past 3 decades, the treatment strategy for patients with renal injuries has shifted from operative management to NOM [9]. Depending on the guideline, conservative treatment is recommended for most patients who are hemodynamically stable. However, conservative management of patients with grade IV or V injuries who are hemodynamically unstable remains controversial [2]. In most high-grade renal injury cases, nephrectomy remains a possibility even though successful NOM is possible [10]. However, recent developments in imaging technology and in the available treatment options have decreased the need for surgical interventions, allowing kidney preservation in most cases [11]. In a recent review, $90 \%$ of 3,528 surveyed patients with renal injuries recovered without open surgical interventions [12]. Altman et al. [13] conservatively treated hemodynamically stable patients with grade $\mathrm{V}$ renal injuries and reported that the intensive care unit stays were shorter, the required blood transfusion amounts were smaller, and the numbers of complications were lower than in patients undergoing surgical interventions.

Renal artery embolization is an effective, minimally invasive treatment for renal injury that can prevent gross hematuria and preserve renal function [11]. According to the 2018 AAST revised grading, active bleeding and vascular injuries (e.g., pseudoaneurysms and arteriovenous fistulas) are included, and interventions may be required in cases of hemodynamic instability [8]. Breyer et al. [14] reported that renal artery embolization was effective in patients with grade IV renal injuries when conservative treatment failed. According to them, the indications for renal artery embolization include renal injuries with persistent gross hematuria, increasing perirenal hematoma, renal arteriovenous fistulas, and pseudoaneurysm formation.

Kidney-related complications (excluding renal failure) occur in approximately $5.2 \%$ of patients with renal injuries [15]. The most common complication is persistent urinary extravasation, resulting in the formation of a urinoma [16]. In both cases above, fluid collection occurred and was treated through PCD insertion. Fluid collection around the kidneys, such as urinoma and hematoma, can be a source of infection. PCD insertion to drain a fluid collection may result in a prolonged hospital stay compared to surgical treatment. Furthermore, simple contrast enhancement on CT does not necessarily mean that kidney function has recovered, so additional imaging tests may be needed to accurately confirm kidney function recovery [17].

The regional trauma center of Wonkwang University Hospital is equipped with a hybrid ER system that includes a continuously staffed, in-house IR team. Therefore, we can very quickly perform arterial embolization, which is the treatment of choice for solid organ (e.g., kidney, spleen, and liver) injuries in the abdominal cavity, even in hemodynamically unstable patients. In our patient with a grade V renal injury, a CT scan was performed 16 minutes after arrival, and arterial embolization was initiated within 31 minutes of presentation. In our patient with a grade IV renal injury, common femoral artery puncture was performed within 8 minutes of the patient's arrival, renal artery embolization was completed within 25 minutes, and lumbar artery embolization was completed within 35 minutes of arrival; an additional CT 
evaluation was performed within 42 minutes of arrival. Thanks to the availability of this hybrid ER system and the IR team, bleeding can be controlled within a very short time. As a result, surgery can be avoided and good results can be obtained, including kidney preservation. However, gathering additional cases and comparing the features of these cases with those of previous patients remains necessary. The features that should be compared among cases include blood transfusion volume, intensive care unit stay duration, total hospital stay duration, and complications. The authors believe that the hybrid ER system with inhouse IR team could contribute to NOM and kidney preservation even in hemodynamically unstable patients with high-grade renal injuries. In the future, a comparative study on the treatment results of the conventional ER and hybrid ER systems is considered to be necessary.

\section{REFERENCES}

1. van der Wilden GM, Velmahos GC, Joseph DK, Jacobs L, Debusk MG, Adams CA, et al. Successful nonoperative management of the most severe blunt renal injuries: a multicenter study of the research consortium of New England Centers for Trauma. JAMA Surg 2013;148:924-31.

2. Chouhan JD, Winer AG, Johnson C, Weiss JP, Hyacinthe LM. Contemporary evaluation and management of renal trauma. Can J Urol 2016;23:8191-7.

3. Santucci RA, McAninch JW. Diagnosis and management of renal trauma: past, present, and future. J Am Coll Surg 2000;191: 443-51.

4. Moore EE, Shackford SR, Pachter HL, McAninch JW, Browner $\mathrm{BD}$, Champion HR, et al. Organ injury scaling: spleen, liver, and kidney. J Trauma 1989;29:1664-6.

5. Chien LC, Vakil M, Nguyen J, Chahine A, Archer-Arroyo K, Hanna TN, et al. The American Association for the Surgery of Trauma Organ Injury Scale 2018 update for computed tomography-based grading of renal trauma: a primer for the emergency radiologist. Emerg Radiol 2020;27:63-73.

6. Kozar RA, Crandall M, Shanmuganathan K, Zarzaur BL, Coburn M, Cribari C, et al. Organ injury scaling 2018 up- date: spleen, liver, and kidney. J Trauma Acute Care Surg 2018;85:1119-22.

7. Santucci RA, Wessells H, Bartsch G, Descotes J, Heyns CF, McAninch JW, et al. Evaluation and management of renal injuries: consensus statement of the renal trauma subcommittee. BJU Int 2004;93:937-54.

8. Hosein M, Paskar D, Kodama R, Ditkofsky N. Coming together: a review of the American Association for the surgery of trauma's updated kidney injury scale to facilitate multidisciplinary management. AJR Am J Roentgenol 2019;213:1091-1099.

9. Kuan JK, Wright JL, Nathens AB, Rivara FP, Wessells H; American Association for the Surgery of Trauma. American Association for the surgery of trauma organ injury scale for kidney injuries predicts nephrectomy, dialysis, and death in patients with blunt injury and nephrectomy for penetrating injuries. J Trauma 2006;60:351-6.

10. Salem MS, Urry RJ, Kong VY, Clarke DL, Bruce J, Laing GL. Traumatic renal injury: five-year experience at a major trauma centre in South Africa. Injury 2020;51:39-44.

11. Vozianov S, Sabadash M, Shulyak A. Experience of renal artery embolization in patients with blunt kidney trauma. Cent European J Urol 2015;68:471-7.

12. McClung CD, Hotaling JM, Wang J, Wessells H, Voelzke BB. Contemporary trends in the immediate surgical management of renal trauma using a national database. J Trauma Acute Care Surg 2013;75:602-6.

13. Altman AL, Haas C, Dinchman KH, Spirnak JP. Selective nonoperative management of blunt grade 5 renal injury. J Urol 2000;164:27-30; discussion 30-1.

14. Breyer BN, McAninch JW, Elliott SP, Master VA. Minimally invasive endovascular techniques to treat acute renal hemorrhage. J Urol 2008;179:2248-52; discussion 2253.

15. Starnes M, Demetriades D, Hadjizacharia P, Inaba K, Best C, Chan L. Complications following renal trauma. Arch Surg 2010;145:377-81; discussion 381-2.

16. Lee YJ, Oh SN, Rha SE, Byun JY. Renal trauma. Radiol Clin North Am 2007;45:581-92.

17. Yao YL, Gao Y. Present situation and research progress of kidney function recoverability evaluation of acute kidney injury patient. Int J Gen Med 2021;14:1919-25. 